\title{
Optical metastability in bulk GaN single crystals
}

\author{
I. K. Shmagin, J. F. Muth, J. H. Lee, and R. M. Kolbas \\ Department of Electrical and Computer Engineering, North Carolina State University, \\ Raleigh, North Carolina 27695-7911
}

C. M. Balkas, Z. Sitar, and R. F. Davis

Department of Material Science and Engineering, North Carolina State University, Raleigh, North Carolina 2769-7907

(Received 21 April 1997; accepted for publication 6 May 1997)

\begin{abstract}
Bulk GaN single crystals were grown from cold pressed GaN powder by sublimation in flowing ammonia. Optical transmission measurements indicated that the absorption coefficient for the transparent samples is $50 \mathrm{~cm}^{-1}$ in the wavelength region from 650 to $400 \mathrm{~nm}$. Optical metastability in bulk GaN crystals was studied through time dependent photoluminescence both at room and liquid-nitrogen temperatures. The observation included decreasing output intensity of the ultraviolet emission attributed to the band edge and increasing output intensity of a new emission band centered at $378 \mathrm{~nm}$ at room temperature. At liquid-nitrogen temperature, the photoinduced emission band consisted of at least one LO-phonon replica of the zero-phonon line centered at 378 $\mathrm{nm}$. The ratio of output intensities of the photoinduced band to the band edge increased by a factor of 10 during $27 \mathrm{~min}$ of exposure time. The photoinduced effect is attributed to the metastable nature of traps in bulk GaN. (C) 1997 American Institute of Physics. [S0003-6951(97)00728-6]
\end{abstract}

$\mathrm{GaN}$ has a tremendous potential in the field of optoelectronics and high-power-high-temperature device applications. The first attempt to grow homoepitaxial GaN on polished bulk GaN single crystalline platelets resulted in a high quality epitaxial layer. ${ }^{1,2}$ The availability of production scale bulk GaN single crystalline substrates would substantially accelerate device development.

Metastability and persistent photoconductivity (PPC) have been extensively studied in bulk GaAs. ${ }^{3,4}$ In intrinsic bulk GaAs, the metastable states can be explained by unintentional oxygen doping and the presence of EL2 defects. ${ }^{3}$ The presence of oxygen in GaAs gives rise to deep levels in the material. ${ }^{5,6}$ The so-called O (or EL2) level is known to have a metastable nature and is responsible for the photoelectric memory effect in oxygen rich GaAs. ${ }^{7,8}$ The properties of light-induced defects in semiconductors were summarized recently. ${ }^{6}$ In particular, donor- and acceptorlike deep levels in $\mathrm{GaAs}$ and $\mathrm{ZnSe}$, respectively, are associated with a large lattice relaxation and are responsible for PPC in these materials. ${ }^{9}$ Recently, there has been a report about metastability and persistent conductivity in $p-{ }^{10}$ and $n$-type ${ }^{11}$ doped $\mathrm{GaN}$, where an impurity associated lattice relaxation mechanism has been suggested to be causing the PPC in the $p$ - and $n$-type GaN. Also, an optical memory effect was reported and associated with oxygen impurities in epitaxially grown GaN and AlN films. ${ }^{12}$

In this letter, we report observations of optical metastability in high quality bulk GaN single crystals. Timedependent photoluminescence results from the bulk GaN single crystals at room [RT $295 \mathrm{~K}$ ] and liquid-nitrogen (77 K) temperatures are reported. At RT, a new emission peak centered at $378 \mathrm{~nm}$ was detected on the long-wavelength shoulder of the output emission spectrum after the sample was illuminated with the excitation light $(280 \mathrm{~nm})$. At $77 \mathrm{~K}$, the photoinduced emission peak centered at $388 \mathrm{~nm}$ was observed after the sample was illuminated for $27 \mathrm{~min}$.

The bulk GaN crystals were grown by sublimation of cold pressed GaN pellets in flowing ammonia atmosphere as described elsewhere. ${ }^{13}$ The crystals are well-faceted hexagonally shaped rods with the $c$ axis along the length of the crystal, as shown in Fig. 1. The average samples are 0.2-0.3 $\mathrm{mm}$ wide and $0.7-1.0 \mathrm{~mm}$ long. The crystals are colorless and transparent to the naked eye. Secondary-ion-mass spectroscopy (SIMS) analysis indicated that the sample had 1, 2, and $5 \times 10^{16} \mathrm{~cm}^{-3}$ concentrations of $[\mathrm{Si}],[\mathrm{C}]$, and $[\mathrm{H}]$, and $3 \times 10^{18} \mathrm{~cm}^{-3}$ of [O], respectively.

A Cary 5E UV-Vis-NIR spectrophotometer was used as the light source for the optical transmission measurements. Preparation for photoluminescence consisted of pressing the single crystals into indium on a heat-conductive copper plug. The samples were oriented with the $c$ axis parallel to the indium surface, so that the pump beam was incident on an $a$ plane. The frequency tripled output $\left(\lambda_{p}=280 \mathrm{~nm} ; 250 \mathrm{fs}\right)$ of a Ti:sapphire laser was used as the excitation source. The high intensity excitation source can produce a large density of light-induced effects during a reasonably short time.

The RT transmission curve is plotted versus wavelength in Fig. 2. The absorption band edge is determined to be at $369 \mathrm{~nm}$ and to have a $10 \mathrm{~nm}$ width. From the transmission data, the absorption coefficient was estimated to be $50 \mathrm{~cm}^{-1}$ over the wavelength region from 650 to $400 \mathrm{~nm}$. Such a small absorption coefficient and narrow width of the absorption band edge imply that the bulk GaN single crystals have a very high optical quality and low optical defect density. The slope of the transmission spectrum in the visible region is due to the scattering from the rough surface of the crystals. The thickness of the crystal, the presence of excitons and impurity levels (band-edge tailing) near the band gap preclude accurate assignment of the band gap by this transmission measurement. Instead, a better estimate of the band-gap energy is given by photoluminescence measurements.

The RT photoluminescence from a sample is shown in Fig. 3. The emission associated with the conduction-to- 


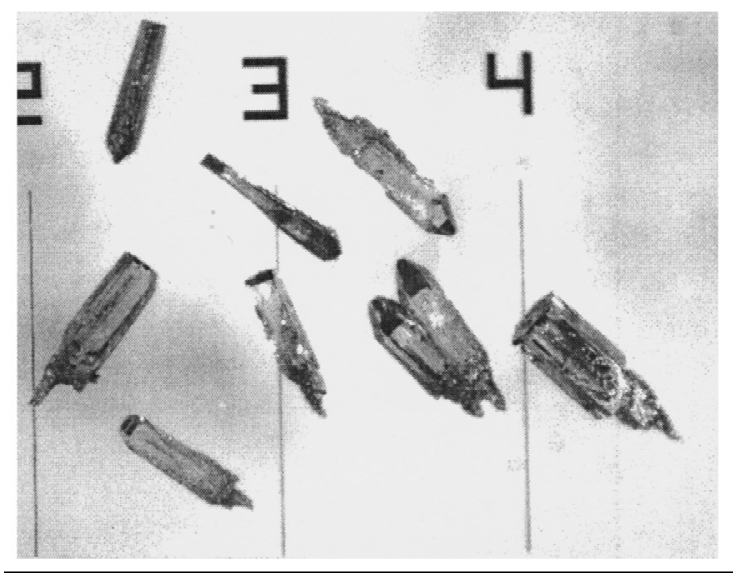

FIG. 1. Bulk GaN single crystals grown by sublimation. The vertical lines are spaced by $1 \mathrm{~mm}$.

valence-band transition is shown in Fig. 3(a). The band-edge attributed emission peak is centered at $365 \mathrm{~nm}$ and has a full width at half-maximum (FWHM) of $13 \mathrm{~nm}(121 \mathrm{meV})$. (The best RT PL spectrum from a bulk GaN sample has a FWHM of $84 \mathrm{meV}$; not shown). No yellow emission attributed to deep donor-to-acceptor transitions was observed in the optical microscope or detected on a scale 500 times more sensitive than that used for the detection of the band edge. The sample was kept illuminated with the excitation light while the spectra were recorded approximately 5 min apart. The emission spectra recorded 5, 9, and 36 min after the first data [Fig. 3(a)] were obtained, are shown in curves (b)-(d) of Fig. 3 , respectively. A new emission peak was detected at $378 \mathrm{~nm}$ on the long-wavelength shoulder of the original emission spectrum after the sample was illuminated for $5 \mathrm{~min}$ [Fig. 3 (b)]. The ratio of the output emission intensities of the longwavelength peak $(378 \mathrm{~nm})$ to the band edge $(365 \mathrm{~nm})$ increased by a factor of 4 during 36 min of exposure time, while the integrated output intensity decreased by a factor of 20. The final emission spectrum shown in Fig. 3(d) has two

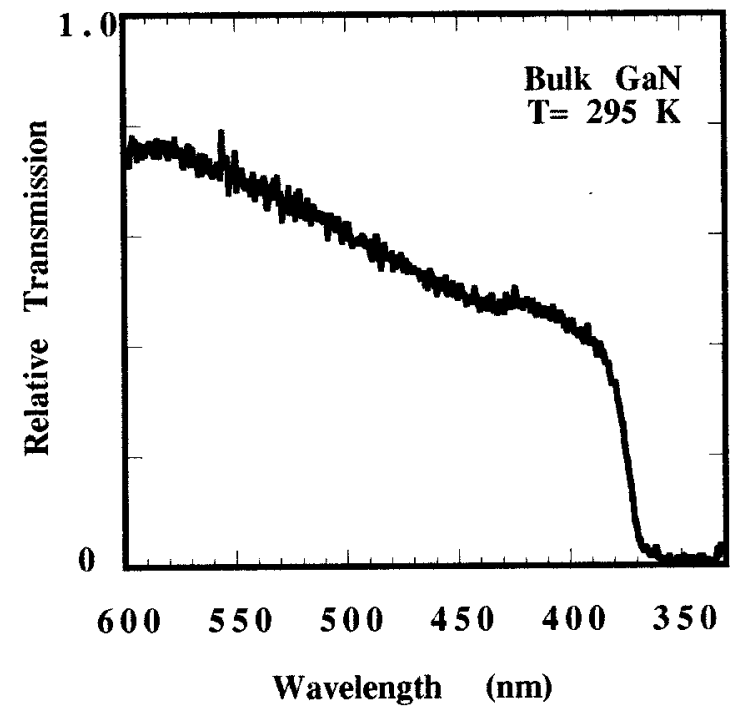

FIG. 2. Room-temperature transmission spectrum from a bulk GaN single crystal. The absorption coefficient is calculated to be $50 \mathrm{~cm}^{-1}$ over the wavelength region from 650 to $400 \mathrm{~nm}$.

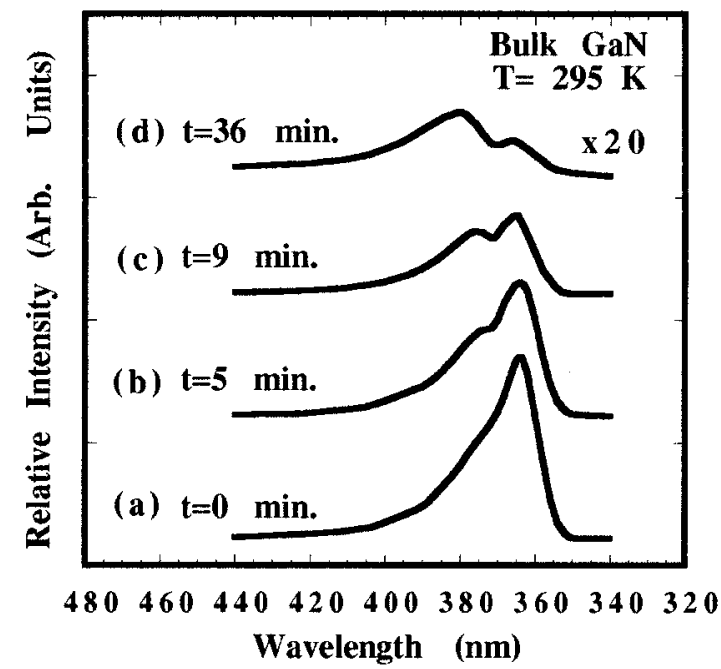

FIG. 3. Time-dependent room-temperature photoluminescence spectra from a bulk GaN single crystal. The output intensity of the photoinduced emission band $(378 \mathrm{~nm})$ increases with the exposure time relative to the band edge $(365 \mathrm{~nm})$.

well-defined emission peaks, one at $365 \mathrm{~nm}$ associated with the band edge and the other centered at $378 \mathrm{~nm}$. The output intensity of the $378 \mathrm{~nm}$ emission peak is higher in magnitude than the band-edge emission [curve 3(d)]. It is possible that the emission band at $378 \mathrm{~nm}$ consists of several overlapping peaks, which are not resolved at RT. The gradual increase in the output emission intensity with longer exposure to the excitation light indicates that the creation and/or filling of trap levels occurs. Traps are metastable energy levels that can capture the carriers and keep them from participating in the radiative recombination process for a significantly long time. ${ }^{14}$

The phenomenon was also studied at liquid-nitrogen temperature. Right after a bulk GaN single crystal was taken to $77 \mathrm{~K}$, the output photoluminescence was usually centered at $359 \mathrm{~nm}$, as shown in Fig. 4(a). It is redshifted $1 \mathrm{~nm}$ relative to the emission-peak wavelength from epitaxially grown $\mathrm{GaN}$ on $c$-plane sapphire, where a peak position of $358 \mathrm{~nm}$ is usually observed. The shift to longer wavelengths for these samples is attributed to a significant reabsorption due to the large thickness of the sample $(0.2 \mathrm{~mm})$. It also could be caused by the lack of stress, which is always present in chemical-vapor-deposition-grown GaN.

After the sample was illuminated for some time, the emission color gradually changed from colorless to bright blue, indicating that the output emission spectrum had changed. The change in spectra of the bulk GaN single crystals is illustrated in Fig. 4. The measurements started with the band-edge emission peak centered at $359 \mathrm{~nm}$ (FWHM $=5 \mathrm{~nm} ; 48 \mathrm{meV}$ ). The spectrum showed a new emission peak centered at $378 \mathrm{~nm}$ after $10 \mathrm{~min}$ of exposure time. The long-wavelength shoulder of the emission peak expands to the visible region and is responsible for the blue color of the output emission. After 27 min of excitation, the blue emission band is centered at $388 \mathrm{~nm}$ and consists of at least one LO-phonon assisted replica of the zero-phonon $(378 \mathrm{~nm})$ line. The energy separation between the peaks is $85 \mathrm{meV}$, which is close to the reported value of $90 \mathrm{meV}$ for a LO 


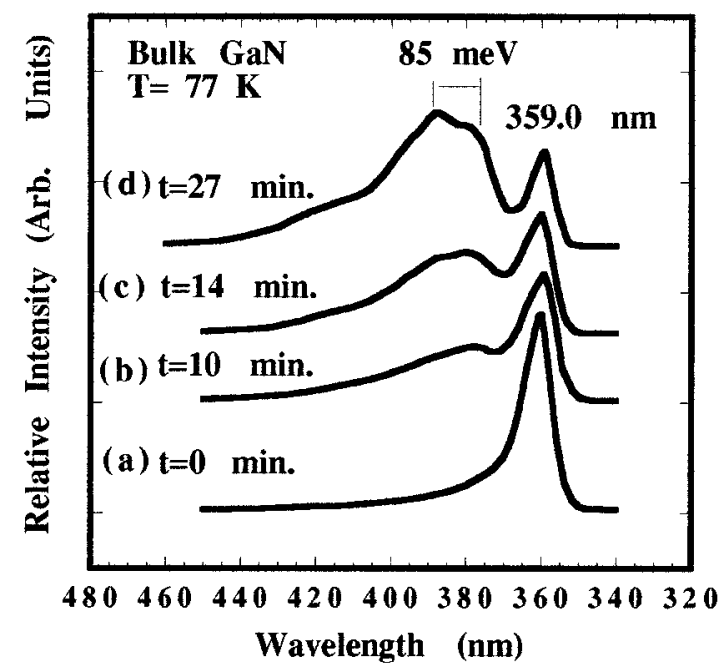

FIG. 4. Time-dependent $77 \mathrm{~K}$ photoluminescence spectra from a bulk GaN single crystal. The photoinduced emission band consists of a zero-phonon line $(378 \mathrm{~nm})$ and a LO-phonon replica of the main peak, as shown in (d). The ratio of blue/UV emission increased by factor 10 during $27 \mathrm{~min}$ of exposure.

phonon in GaN. ${ }^{15,16}$ The FWHM of the blue emission was measured to be $249 \mathrm{meV}$. The significant spectral width of the phonon assisted recombination implies a strong lattice interaction. No decrease in the total output emission intensity was detected at $77 \mathrm{~K}$, while the ratio of blue $(388 \mathrm{~nm})$ to UV $(359 \mathrm{~nm})$ output intensities increased by a factor of 10 during 27 min of exposure. After the last spectrum was recorded, the excitation was terminated and the sample was left inside the cryogenic dewar at $77 \mathrm{~K}$ in a dark room for $45 \mathrm{~min}$ more. Then, the excitation source was opened again and another spectrum was taken and found to be the same as the spectra of Fig. 4(d).

No physical damage was introduced to the surface of the sample as observed under the optical microscope. To verify that the change in the optical properties was indeed metastable, the room-temperature emission spectrum was taken from the sample two weeks after the initial results were obtained. The integrated output emission was restored to its original intensity, but the blue emission band was still clearly observed. Such a long recovery time indicates that the photoinduced levels have a large thermal activation energy or that the process is not reversible.

The PL results presented above are consistent with the creation and/or filling of a metastable state in the bulk GaN single crystals due to the high intensity irradiation by the pump source. The $77 \mathrm{~K} \mathrm{PL}$ data indicate that the photoinduced radiative recombination is assisted by LO phonons. The energy of the LO phonons was estimated to be $85 \mathrm{meV}$ from the $77 \mathrm{~K}$ PL results. It is possible that the photoinduced emission band consists of several overlapping peaks of different origin, which are not resolved in the present measure- ments. In $n$-type $\mathrm{GaN}$, effects originating from the presence of Ga vacancies should be predominant. ${ }^{17} \mathrm{We}$ propose that the photoinduced emission band reported above is formed by a superposition of LO-phonon assisted radiative transitions from the conduction band to the acceptor and from a shallow donor formed by oxygen to the same acceptor. Our experimental results are consistent with a presence of nonradiative metastable levels in the GaN forbidden gap. These levels compete with the band-edge recombination and prevent the observation of the donor-related emission in the uncharged state. After the metastable levels are filled, they do not participate in the recombination process, which results in an increase in the integrated output emission intensity.

In summary, we report a photoluminescence study of optical metastability in bulk GaN single crystals. The observations included time dependent PL in which a photoinduced emission band was observed on the long-wavelength shoulder of the emission peak at RT and $77 \mathrm{~K}$. The photoinduced emission band is attributed to a superposition of the LOphonon assisted free-electron-to-bound-hole and donor-todeep-acceptor radiative transitions. The increase in the integrated output emission intensity as a function of exposure time to the excitation light indicated the presence of traps inside the material.

This work was supported by the U.S. Army Research Office under Contract No. DAAH04-93-D-003-4 supervised by Dr. John Zavada, and the Office of Naval Research under Contract No. N00014-92-J-1477.

${ }^{1}$ F. A. Ponce, D. P. Bour, W. Götz, N. M. Johnson, H. I. Helava, I. Grzegory, J. Jun, and S. Porowski, Appl. Phys. Lett. 68, 917 (1996).

${ }^{2}$ S. Porowski, J. Cryst. Growth 166, 583 (1996).

${ }^{3}$ P. W. Yu, Solid State Commun. 43, 953 (1982).

${ }^{4}$ M. Tajima, in Defects and Properties of Semiconductors: Defect Engineering, edited by J. Chikawa, K. Sumino, and K. Wada (KTK Scientific, 1987), pp. 37-69.

${ }^{5}$ G. H. Gooch, C. Hilsum, and R. B. Holeman, J. Appl. Phys. 32, 2069 (1961).

${ }^{6}$ D. Redfield and R. H. Bube, Photoinduced Defects in Semiconductors (Cambridge University Press, Cambridge, 1996).

${ }^{7}$ G. Vincent, D. Bois, and A. Chantre, J. Appl. Phys. 53, 3643 (1982).

${ }^{8}$ C. H. Henry and D. V. Lang, Phys. Rev. B 15, 989 (1977).

${ }^{9}$ D. Redfield and R. H. Bube, Photoinduced Defects in Semiconductors (Cambridge University Press, Cambridge, 1996), p. 12.

${ }^{10}$ C. Johnson, J. Y. Lin, H. X. Jiang, M. Asif Khan, and C. J. Sun, Appl. Phys. Lett. 68, 1808 (1996).

${ }^{11}$ A. E. Wickenden, G. Beadie, D. D. Koleske, W. S. Rabinovich, and J. A. Freitas, Jr., Mater. Res. Soc. Symp. Proc. 449, 531.

${ }^{12}$ V. A. Joshkin, J. C. Roberts, E. L. Piner, M. K. Behbehani, F. G. McIntosh, and S. M. Bedair, Appl. Phys. Lett. 71, 234 (1997).

${ }^{13}$ C. M. Balkas, Z. Sitar, T. Zheleva, L. Bergman, I. K. Shmagin, J. F. Muth, R. M. Kolbas, R. Nemanich, and R. F. Davis, Mater. Res. Soc. Symp. Proc. 449, 41.

${ }^{14}$ J. I. Pankove, in Optical Processes in Semiconductors (Dover, New York, 1971), p. 370.

${ }^{15}$ D. D. Manchon, Jr., A. S. Barker, Jr., P. J. Dean, and R. B. Zetterstorm, Solid State Commun. 8, 1227 (1970).

${ }^{16}$ R. Dingle and M. Ilegems, Solid State Commun. 9, 175 (1971).

${ }^{17}$ J. Neugebauer and C. G. Van de Walle, Appl. Phys. Lett. 69, 503 (1996). 